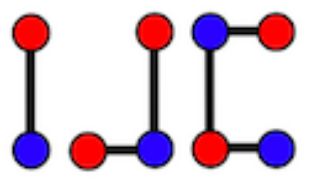

\title{
Total edge irregularity strength of some cycle related graphs
}

\author{
R. Ramalakshmi ${ }^{\text {a }}$, K.M. Kathiresan ${ }^{\text {b }}$ \\ 'Department of Mathematics, Rajapalayam Rajus' College, Rajapalayam -626 117, India. \\ ${ }^{b}$ Centre for Graph Theory, Ayya Nadar Janaki Ammal College, Sivakasi-626 124, India. \\ ramimani20@gmail.com, kathir2esan@yahoo.com
}

\begin{abstract}
An edge irregular total $k$-labeling $f: V \cup E \rightarrow\{1,2, \ldots, k\}$ of a graph $G=(V, E)$ is a labeling of vertices and edges of $G$ in such a way that for any two different edges $u v$ and $u^{\prime} v^{\prime}$, their weights $f(u)+f(u v)+f(v)$ and $f\left(u^{\prime}\right)+f\left(u^{\prime} v^{\prime}\right)+f\left(v^{\prime}\right)$ are distinct. The total edge irregularity strength tes $(G)$ is defined as the minimum $k$ for which the graph $G$ has an edge irregular total $k$-labeling. In this paper, we determine the total edge irregularity strength of new classes of graphs $C_{m} @ C_{n}, P_{m, n}^{*}$ and $C_{m, n}^{*}$ and hence we extend the validity of the conjecture tes $(G)=\max \left\{\left\lceil\frac{|E(G)|+2}{3}\right\rceil,\left\lceil\frac{\Delta(G)+1}{2}\right\rceil\right\}$
\end{abstract} for some more graphs.

Keywords: edge irregularity strength, total edge irregularity strength

Mathematics Subject Classification: 05C78

DOI: $10.19184 /$ ijc.2021.5.1.3

\section{Introduction}

Throughout this paper, $G$ is a simple graph, $V$ and $E$ are the sets of vertices and edges of $G$, with cardinalities $|V|$ and $|E|$ respectively. A labeling of a graph is a map that carries graph elements to the numbers. A labeling is called a vertex labeling, an edge labeling or a total labeling, if the domain of the map is the vertex set, the edge set, or the union of vertex and edge sets respectively.

Received: 29 February 2020, Revised: 16 April 2020, Accepted: 26 December 2020. 
Baca et al. in [2] started to investigate the total edge irregularity strength of a graph, an invariant analogous to the irregularity strength for total labeling. For a graph $G=(V(G), E(G))$, the weight of an edge $e=x y$ under a total labeling $\xi$ is $w t_{\xi}(e)=\xi(x)+\xi(e)+\xi(y)$. For a graph $G$ we define a labeling $\xi: V(G) \cup E(G) \rightarrow\{1,2, \cdots, k\}$ to be an edge irregular total $k$-labeling of the graph $G$ if for every two different edges $x y$ and $x^{\prime} y^{\prime}$ of $G$ one has $w t_{\xi}(x y) \neq w t_{\xi}\left(x^{\prime} y^{\prime}\right)$. The total edge irregular strength, tes $(G)$, is defined as the minimum $k$ for which $G$ has an edge irregular total $k$-labeling. In [3], we can find that

$$
\operatorname{tes}(G) \geq \max \left\{\left\lceil\frac{|E(G)|+2}{3}\right\rceil,\left\lceil\frac{\Delta(G)+1}{2}\right\rceil\right\},
$$

where $\Delta(G)$ is the maximum degree of $G$, and also there are determined the exact values of the total edge irregularity strength for paths, cycles, stars, wheels and friendship graphs. Recently Ivanco and Jendrol [6] proved that for any tree $T$,

$$
\operatorname{tes}(T)=\max \left\{\left\lceil\frac{|E(G)|+2}{3}\right\rceil,\left\lceil\frac{\Delta(G)+1}{2}\right\rceil\right\} .
$$

Moreover, they posed a conjecture that for an arbitrary graph $G$ different from $K_{5}$ and the maximum degree $\Delta(G)$,

$$
\operatorname{tes}(G)=\max \left\{\left\lceil\frac{|E(G)|+2}{3}\right\rceil,\left\lceil\frac{\Delta(G)+1}{2}\right\rceil\right\} .
$$

The Ivanco and Jendrol's conjecture has been verified for complete graphs and complete bipartite graphs in [7], for categorical product of cycle and path in [1] and [12], for corona product of paths with some graphs in [11]. In[8], Jeyanthi et al. verified the conjecture for disjoint union of double wheel graphs. In[5], Indra et al. verified the conjecture for generalized uniform theta graph.

Motivated by the papers $[9,10]$, we define three new classes of graphs and extend the validity for the conjecture for some more families of graphs. We define the graph $C_{m} @ C_{n}, n \geq 3, m \geq 3$ as follows. Denote the vertex set of $C_{m} @ C_{n}$ by $V\left(C_{m} @ C_{n}\right)=\left\{u_{i} \mid 1 \leq i \leq n\right\} \cup\left\{v_{i j} \mid 1 \leq i \leq\right.$ $n, 1 \leq j \leq m\}$ and the edge set of $C_{m} @ C_{n}$ by $E\left(C_{m} @ C_{n}\right)=\left\{e_{i}=u_{i} u_{i+1} \mid 1 \leq i \leq n, u_{n+1}=\right.$ $\left.u_{1}\right\} \cup\left\{e_{i 1}=u_{i} v_{i 1} \mid 1 \leq i \leq n\right\} \cup\left\{e_{i(j+1)}=v_{i j} v_{i(j+1)} \mid 1 \leq i \leq n, 1 \leq j \leq m, v_{i(m+1)}=v_{i 1}\right\}$. In $C_{m} @ C_{n},\left|V\left(C_{m} @ C_{n}\right)\right|=n(m+1)$ and $\left|E\left(C_{m} @ C_{n}\right)\right|=n(m+2)$. The graph $C_{3} @ C_{9}$ is shown in Figure 1.

We introduce another new class of graph $P_{m, n}^{*}$. The graph $P_{m, n}^{*}, m \geq 3, n \geq 2$ is defined as follows: denote the vertex set of $P_{m, n}^{*}$ by $V\left(P_{m, n}^{*}\right)=\left\{v_{i} \mid 1 \leq i \leq n+1\right\} \cup\left\{v_{i j} \mid 1 \leq i \leq\right.$ $n, 1 \leq j \leq m-2\}$ and the edge set of $P_{m, n}^{*}$ by $E\left(P_{m, n}^{*}\right)=\left\{e_{i}=v_{i} v_{i+1} \mid 1 \leq i \leq n\right\} \cup\left\{e_{i j}=\right.$ $\left.v_{i(j-1)} v_{i j} \mid 1 \leq i \leq n, 2 \leq j \leq m-2\right\} \cup\left\{e_{i 1}=v_{i} v_{i 1)} \mid 1 \leq i \leq n\right\} \cup\left\{e_{i(m-1)}=v_{i(m-2)} v_{i 1} \mid 1 \leq i \leq\right.$ $n\}$. In $P_{m, n}^{*},\left|V\left(P_{m, n}^{*}\right)\right|=m n-n+1$ and $\left|E\left(P_{m, n}^{*}\right)\right|=n m$. The graph $P_{4,7}^{*}$ is shown in Figure 2. In $P_{m, n}^{*}, m \geq 3, n \geq 2$, identifying the vertices $v_{1}$ and $v_{n+1}$ we obtain the new class of graph denoted by $C_{m, n}^{*}$. 
In this paper, we determine the total edge irregularity strength of these new classes of graphs $C_{m} @ C_{n}, P_{m, n}^{*}$ and $C_{m, n}^{*}$ and hence we extend the validity of the conjecture

$$
\operatorname{tes}(G)=\max \left\{\left\lceil\frac{|E(G)|+2}{3}\right\rceil,\left\lceil\frac{\Delta(G)+1}{2}\right\rceil\right\}
$$

for some more families of graphs.

\section{Main Results}

In the following theorem we describe an optimal edge irregular total labeling for the graph $C_{m} @ C_{n}$.

Theorem 2.1. For any integers $m \geq 3, n \geq 3$, tes $\left(C_{m} @ C_{n}\right)=\left\lceil\frac{n(m+2)+2}{3}\right\rceil$.

Proof. The vertex set of $C_{m} @ C_{n}$ is $\left\{u_{i} \mid 1 \leq i \leq n\right\} \cup\left\{v_{i j} \mid 1 \leq i \leq n, 1 \leq j \leq m\right\}$ and the edge set of $C_{m} @ C_{n}$ is $\left\{e_{i}=u_{i} u_{i+1} \mid 1 \leq i \leq n, u_{n+1}=u_{1}\right\} \cup\left\{e_{i 1}=u_{i} v_{i 1} \mid 1 \leq i \leq n\right\} \cup\left\{e_{i(j+1)}=\right.$ $\left.v_{i j} v_{i(j+1)} \mid 1 \leq i \leq n, 1 \leq j \leq m, v_{i(m+1)}=v_{i 1}\right\}$. Since $\operatorname{tes}(G) \geq \max \left\{\left\lceil\frac{|E(G)|+2}{3}\right\rceil,\left\lceil\frac{\Delta(G)+1}{2}\right\rceil\right\}$, it is enough to prove that $\operatorname{tes}\left(C_{m} @ C_{n}\right) \leq\left\lceil\frac{n(m+2)+2}{3}\right\rceil$.

Let $k=\left\lceil\frac{n(m+2)+2}{3}\right\rceil$.

We construct an edge-irregular total labeling $l$ as follows:

$$
\begin{aligned}
& l\left(u_{i}\right)= \begin{cases}1 & \text { if } i=1 \\
\left\lceil\frac{m+3}{2}\right\rceil & \text { if } i=2 \\
m+5 & \text { if } i=3 \\
k-\left(\left\lfloor\frac{n-6}{2}\right\rfloor-(i-3) m\right) & \text { if } 4 \leq i<\left\lfloor\frac{n}{2}\right\rfloor \\
k & \text { if }\left\lfloor\frac{n}{2}\right\rfloor \leq i \leq\left\lfloor\frac{n}{2}\right\rfloor+2 \\
k-\left\lfloor\frac{i-\left\lfloor\frac{n}{2}\right\rfloor}{2}\right\rfloor m & \text { if }\left\lfloor\frac{n}{2}\right\rfloor+3 \leq i \leq n-2 \\
\left\lceil\frac{3 m+7}{2}\right\rceil & \text { if } i=n-1 \\
3+m & \text { if } i=n,\end{cases} \\
& l\left(v_{1 j}\right)=\left\lfloor\frac{j+4}{3}\right\rfloor \text { for } 1 \leq j \leq m, \\
& l\left(v_{2 j}\right)=\left\lfloor\frac{m+j+5}{3}\right\rfloor \text { for } 1 \leq j \leq m \text {, } \\
& l\left(v_{i j}\right)= \begin{cases}\left\lfloor\frac{m+3+(2 i-3)(m+2)}{3}\right\rfloor & \text { for } 1 \leq j \leq m, 3 \leq i \leq\left\lceil\frac{n}{2}\right\rceil \\
\left\lceil\frac{m+4+(n-1)(m+2)}{3}\right\rceil & \text { for } 1 \leq j \leq m, i=\left\lceil\frac{n}{2}\right\rceil+1 \\
\left\lfloor\frac{m+3+(2 n-2 i+2)(m+2)}{3}\right\rfloor & \text { for } 1 \leq j \leq m,\left\lceil\frac{n}{2}\right\rceil+2 \leq i \leq n,\end{cases}
\end{aligned}
$$




$$
l\left(e_{i}\right)= \begin{cases}m+3-\left\lceil\frac{m+3}{2}\right\rceil & \text { if } i=1 \\ 2 m+3-\left\lceil\frac{m+3}{2}\right\rceil & \text { if } i=2 \\ 3 m+7-k+\left\lfloor\frac{n-6}{2}\right\rfloor m= & \text { if } i=3 \\ 4 m+4 i-2 k+2\left\lfloor\frac{n-6}{2}\right\rfloor m & \text { if } 4 \leq i<\left\lfloor\frac{n}{2}\right\rfloor-1 \\ m+\left\lfloor\frac{n}{2}\right\rfloor m+\left\lfloor\frac{n-6}{2}\right\rfloor m+4\left\lfloor\frac{n}{2}\right\rfloor-4-2 k & \text { if } i=\left\lfloor\frac{n}{2}\right\rfloor-1 \\ 2\left\lfloor\frac{n}{2}\right\rfloor m+4\left\lfloor\frac{n}{2}\right\rfloor-m-2 k & \text { if } i=\left\lfloor\frac{n}{2}\right\rfloor \\ m n+2 n-m+1-2 k & \text { if } i=\left\lfloor\frac{n}{2}\right\rfloor+1 \\ 2 m n-m+4 n-2\left\lfloor\frac{n}{2}\right\rfloor m-4\left\lfloor\frac{n}{2}\right\rfloor-2 k-2 & \text { if } i=\left\lfloor\frac{n}{2}\right\rfloor+2 \\ 2 m n+4 n+2 m+2 i m-4 i+6-2 k-\left\lfloor\frac{i-\left\lfloor\frac{n}{2}\right\rfloor}{2}\right\rfloor m+\left\lfloor\frac{i+1-\left\lfloor\frac{n}{2}\right\rfloor}{2}\right\rfloor m & \text { if }\left\lfloor\frac{n}{2}\right\rfloor+3 \leq i \leq n-3 \\ 6 m+14-k+\left\lfloor\frac{n-2-\left\lfloor\frac{n}{2}\right\rfloor}{2}\right\rfloor m-\left\lceil\frac{3 m+7}{2}\right\rceil & \text { if } i=n-2 \\ 3 m+7-\left\lceil\frac{3 m+7}{2}\right\rceil & \text { if } i=n-1 \\ m+2 & \text { if } i=n,\end{cases}
$$

$l\left(e_{1 j}\right)= \begin{cases}1 & \text { if } j=1 \\ j+2-\left\lfloor\frac{j+3}{3}\right\rfloor-\left\lfloor\frac{j+4}{3}\right\rfloor & \text { if } 2 \leq j \leq m \\ m+2-\left\lfloor\frac{m+4}{3}\right\rfloor & \text { if } j=m+1\end{cases}$

$l\left(e_{i j}\right)= \begin{cases}j+2+(2 i-3)(m+2)-2\left\lfloor\frac{(m+3)+(2 i-3)(m+2)}{3}\right\rfloor & \text { if } 2 \leq j \leq m+1,3 \leq i \leq\left\lceil\frac{n}{2}\right\rceil \\ j+3+(n-1)(m+2)-2\left\lceil\frac{m+4+(n-1)(m+2)}{3}\right\rceil & \text { if } 2 \leq j \leq m+1, i=\left\lceil\frac{n}{2}\right\rceil+1 \\ j+2+(2 n-2 i+2)(m+2)-2\left\lfloor\frac{(m+3)+(2 n-2 i+2)(m+2)}{3}\right\rfloor & \text { if } 2 \leq j \leq m+1,\left\lceil\frac{n}{2}\right\rceil+2 \leq i \leq n,\end{cases}$

$l\left(e_{2 j}\right)=\left\{\begin{array}{cl}m+4+j-\left\lfloor\frac{m+4+j}{3}\right\rfloor-\left\lfloor\frac{m+5+j}{3}\right\rfloor & \text { if } 2 \leq j \leq m \\ 2 m+5-\left\lfloor\frac{2 m+5}{3}\right\rfloor-\left\lfloor\frac{m+6}{3}\right\rfloor & \text { if } j=m+1 .\end{array}\right.$

For $n$ is odd,

$$
l\left(e_{i 1}\right)= \begin{cases}m+5-\left\lfloor\frac{m+6}{3}\right\rfloor-\left\lceil\frac{m+3}{2}\right\rceil & \text { if } i=2 \\ 2 m+4-\left\lfloor\frac{4 m+9}{3}\right\rfloor & \text { if } i=3 \\ m i+4 i-3-k+\left\lfloor\frac{n-6}{2}\right\rfloor m-\left\lfloor\frac{m+3+(2 i-3)(m+2)}{3}\right\rfloor & \text { if } 4 \leq i<\left\lfloor\frac{n}{2}\right\rfloor \\ 2 m i+4 i-3 m-3-k-\left\lfloor\frac{m+3+(2 i-3)(m+2)}{3}\right\rfloor & \text { if }\left\lfloor\frac{n}{2}\right\rfloor \leq i \leq\left\lfloor\frac{n}{2}\right\rfloor+1 \\ 4+(n-1)(m+2)-k-\left\lceil\frac{m+4+(n-1)(m+2)}{3}\right\rceil & \text { if } i=\left\lfloor\frac{n}{2}\right\rfloor+2 \\ 3+(2 n-2 i+2)(m+2)-k+\left\lfloor\frac{i-\left\lfloor\frac{n}{2}\right\rfloor}{2}\right\rfloor m-\left\lfloor\frac{m+3+(2 n-2 i+2)(m+2)}{3}\right\rfloor & \text { if }\left\lfloor\frac{n}{2}\right\rfloor+3 \leq i \leq n-2 \\ 4 m+11-\left\lceil\frac{3 m+7}{2}\right\rceil-\left\lfloor\frac{m+3+4(m+2)}{3}\right\rfloor & \text { if } i=n-1 \\ m+4-\left\lfloor\frac{3 m+7}{3}\right\rfloor & \text { if } i=n .\end{cases}
$$

For $n$ is even,

$$
l\left(e_{i 1}\right)= \begin{cases}m+5-\left\lfloor\frac{m+6}{3}\right\rfloor-\left\lceil\frac{m+3}{2}\right\rceil & \text { if } i=2 \\ 2 m+4-\left\lfloor\frac{4 m+9}{3}\right\rfloor & \text { if } i=3 \\ m i+4 i-3-k+\left\lfloor\frac{n-6}{2}\right\rfloor m-\left\lfloor\frac{m+3+(2 i-3)(m+2)}{3}\right\rfloor & \text { if } 4 \leq i<\frac{n}{2} \\ 2 m i+4 i-3 m-3-k+\left\lfloor\frac{m+3+(2 i-3)(m+2)}{3}\right\rfloor & \text { if } i=\frac{n}{2} \\ 4+(n-1)(m+2)-k-\left\lceil\frac{m+4+(n-1)(m+2)}{3}\right\rceil & \text { if } i=\frac{n}{2}+1 \\ 3+(2 n-2 i+2)(m+2)-k-\left\lfloor\frac{i-\left\lfloor\frac{n}{2}\right\rfloor}{2}\right\rfloor m-\left\lfloor\frac{m+3+(2 n-2 i+2)(m+2)}{3}\right\rfloor & \text { if } \frac{n}{2}+2 \leq i \leq n-2 \\ 4 m+11-\left\lceil\frac{3 m+7}{2}\right\rceil-\left\lfloor\frac{m+3=4(m+2)}{3}\right\rfloor & \text { if } i=n-1 \\ m+4-\left\lfloor\frac{3 m+7}{3}\right\rfloor & \text { if } i=n .\end{cases}
$$

Now $\max \left\{\left\{l(v) \mid v \in V\left(C_{m} @ C_{n}\right)\right\} \cup\left\{l(e) \mid e \in E\left(C_{m} @ C_{n}\right)\right\}\right\}=k$ and $l$ is a function 
from $V\left(C_{m} @ C_{n}\right) \cup E\left(C_{m} @ C_{n}\right)$ into $\{1,2, \ldots, k\}$.

The weights of the edges are given by

$$
\begin{gathered}
w\left(e_{i j}\right)= \begin{cases}m+4 & \text { if } i=1 \\
2+(2 i-1)(m+2) & \text { if } 2 \leq i \leq\left\lceil\frac{n}{2}\right\rceil \\
3+(n-1)(m+2) & \text { if } i=\left\lceil\frac{n}{2}\right\rceil+1 \\
2+(2 n-2 i+2)(m+2) & \text { if }\left\lceil\frac{n}{2}\right\rceil+2 \leq i \leq n,\end{cases} \\
w\left(e_{i}\right)= \begin{cases}j+2 & \text { if } i=1 \\
j+2+(2 i-3)(m+2) & \text { if } 2 \leq i \leq\left\lfloor\frac{n}{2}\right\rfloor \\
j+3+(n-1)(m+2) & \text { if } i=\left\lfloor\frac{n}{2}\right\rfloor+1 \\
j+2+(2 n-2 i+2)(m+2) & \text { if }\left\lfloor\frac{n}{2}\right\rfloor+2 \leq i \leq n .\end{cases}
\end{gathered}
$$

The weights of the edges of $E$ under total labeling $l$ form a set of consecutive integers from 3 to $n(m+2)+2$ and no two edges have the same weight. Hence tes $\left(C_{m} @ C_{n}\right)=\left\lceil\frac{n(m+2)+2}{3}\right\rceil$.

In the following theorem we describe an optimal edge irregular total labeling for the graph $P_{m, n}^{*}$.

Theorem 2.2. For any integers $m \geq 3, n \geq 2$, tes $\left(P_{m, n}^{*}\right)=\left\lceil\frac{n m+2}{3}\right\rceil$.

Proof. The vertex set of $P_{m, n}^{*}$ is $\left\{v_{i} \mid 1 \leq i \leq n+1\right\} \cup\left\{v_{i j} \mid 1 \leq i \leq n, 1 \leq j \leq m-2\right\}$ and the edge set of is $\left\{e_{i}=v_{i} v_{i+1} \mid 1 \leq i \leq n\right\} \cup\left\{e_{i j}=v_{i(j-1)} v_{i j} \mid 1 \leq i \leq n, 2 \leq j \leq\right.$ $m-2\} \cup\left\{e_{i 1}=v_{i} v_{i 1} \mid 1 \leq i \leq n\right\} \cup\left\{e_{i(m-1)}=v_{i(m-2)} v_{i+1} \mid 1 \leq i \leq n\right\}$. Since tes $(G)$ $\geq \max \left\{\left\lceil\frac{|E(G)|+2}{3}\right\rceil,\left\lceil\frac{\Delta(G)+1}{2}\right\rceil\right\}$, it is enough to prove that $\operatorname{tes}\left(P_{m, n}^{*}\right) \leq\left\lceil\frac{n m+2}{3}\right\rceil$.

We construct an edge-irregular total labeling $l$ as follows:

$$
\begin{aligned}
& l\left(v_{i}\right)= \begin{cases}1 & \text { if } i=1, i=n+1 \\
\left\lfloor\frac{\left.\frac{m+5}{3}\right\rfloor}{\left\lfloor\frac{(2 i-2) m+1}{3}\right\rfloor}\right. & \text { if } i=2 \\
\left\lceil\frac{n m+2}{3}\right\rceil & \text { if } 3 \leq i \leq\left\lfloor\frac{n+2}{2}\right\rfloor \\
\left\lfloor\frac{(2 n-2 i+5) m+1}{3}\right\rfloor & \text { if } i=\left\lfloor\frac{n+2}{2}\right\rfloor+1,\left\lfloor\frac{n+2}{2}\right\rfloor+2 \\
\text { if }\left\lfloor\frac{n+2}{2}\right\rfloor+3 \leq i \leq n,\end{cases} \\
& l\left(v_{i j}\right)= \begin{cases}\left\lfloor\frac{j+2}{3}\right\rfloor & \text { if } i=1,1 \leq j \leq m-2 \\
\left\lfloor\frac{m+5}{3}\right\rfloor & \text { if } i=2,1 \leq j \leq m-2 \\
\left\lfloor\frac{(2 i-2) m+1}{3}\right\rfloor & \text { if } 3 \leq i \leq\left\lfloor\frac{n+2}{2}\right\rfloor, 1 \leq j \leq m-2 \\
\left\lfloor\frac{(2 n-2 i+3) m+1}{3}\right\rfloor & \text { if }\left\lfloor\frac{n+2}{2}\right\rfloor+1 \leq i \leq n, 1 \leq j \leq m-2\end{cases} \\
& l\left(e_{i}\right)= \begin{cases}m+1-\left\lfloor\frac{m+5}{3}\right\rfloor & \text { if } i=1 \\
2 m+2-\left\lfloor\frac{m+5}{3}\right\rfloor-\left\lfloor\frac{4 m+1}{3}\right\rfloor & \text { if } i=2 \\
2 i m-2 m+2-\left\lfloor\frac{(2 i-2) m+1}{3}\right\rfloor-\left\lfloor\frac{2 i m+1}{3}\right\rfloor & \text { if } 3 \leq i \leq\left\lfloor\frac{n+2}{2}\right\rfloor-1 \\
\left(2\left\lfloor\frac{n+2}{2}\right\rfloor-4\right) m+2-\left\lceil\frac{m n+2}{3}\right\rfloor-\left\lfloor\frac{\left(2\left\lfloor\frac{n+2}{2}\right\rfloor-4\right) m+1}{3}\right\rfloor & \text { if } i=\left\lfloor\frac{n+2}{2}\right\rfloor \\
\left(2 n-2\left\lfloor\frac{n+2}{2}\right\rfloor+1\right) m+2-\left\lfloor\frac{\left(2 n-2\left\lfloor\frac{n+2}{2}\right\rfloor+1\right) m+1}{3}\right\rfloor & \text { if } i=\left\lfloor\frac{n+2}{2}\right\rfloor+1 \\
(2 n-2 i+3) m+1-\left\lfloor\frac{(2 n-2 i+5) m+1}{3}\right\rfloor-\left\lfloor\frac{(2 n-2 i+3) m+1}{3}\right\rfloor & \text { if }\left\lfloor\frac{n+2}{2}\right\rfloor+2 \leq i \leq n,\end{cases}
\end{aligned}
$$




$$
\begin{aligned}
& l\left(e_{i 1}\right)= \begin{cases}1 & \text { if } i=1 \\
m+3-2\left\lfloor\frac{m+5}{3}\right\rfloor & \text { if } i=2 \\
(2 i-3) m+3-2\left\lfloor\frac{(2 i-2) m+1}{3}\right\rfloor & \text { if } 3 \leq i \leq\left\lfloor\frac{n+2}{2}\right\rfloor \\
\left(2 n-2\left\lfloor\frac{n+2}{2}\right\rfloor\right) m+m+1-\left\lceil\frac{m n+2}{3}\right\rceil-\left\lfloor\frac{\left(2 n-2\left\lfloor\frac{n+2}{2}\right\rfloor+1\right) m+1}{3}\right\rfloor & \text { if } i=\left\lfloor\frac{n+2}{2}\right\rfloor+1 \\
\left(2 n-2\left\lfloor\frac{n+2}{2}\right\rfloor-2\right) m+m+1-\left\lceil\frac{m n+2}{3}\right\rceil-\left\lfloor\frac{\left(2 n-2\left\lfloor\frac{n+2}{2}\right\rfloor-1\right) m+1}{3}\right\rfloor & \text { if } i=\left\lfloor\frac{n+2}{2}\right\rfloor+2 \\
(2 n-2 i+2) m+m+1-\left\lfloor\frac{(2 n-2 i+3) m+1}{3}\right\rfloor-\left\lfloor\frac{(2 n-2 i+1) m+1}{3}\right\rfloor & \text { if }\left\lfloor\frac{n+2}{2}\right\rfloor+3 \leq i \leq n,\end{cases} \\
& l\left(e_{i j}\right)= \begin{cases}m+1-\left\lfloor\frac{m}{3}\right\rfloor-\left\lfloor\frac{m+5}{3}\right\rfloor & \text { if } i=1, j=m-1 \\
j+2-\left\lfloor\frac{j+2}{3}\right\rfloor-\left\lfloor\frac{j+1}{3}\right\rfloor & \text { if } i=1,2 \leq j \leq m-2 \\
m+2+j-2\left\lfloor\frac{m+5}{3}\right\rfloor & \text { if } i=2,2 \leq j \leq m-2 \\
2 m+1-\left\lfloor\frac{m+5}{3}\right\rfloor-\left\lfloor\frac{4 m+1}{3}\right\rfloor & \text { if } i=2, j=m-1 \\
(2 i-3) m+2+j-2\left\lfloor\frac{(2 i-2) m+1}{3}\right\rfloor & \text { if } 3 \leq i \leq\left\lfloor\frac{n+2}{2}\right\rfloor-2,2 \leq j \leq m-2 \\
(2 i-3) m+m+1-\left\lfloor\frac{(2 i-2) m+1}{3}\right\rfloor-\left\lfloor\frac{n m+2}{3}\right\rceil & \text { if } i=\left\lfloor\frac{n+2}{2}\right\rfloor-1,\left\lfloor\frac{n+2}{2}\right\rfloor, 2 \leq j \leq m-2 \\
(2 i-3) m+m+1-\left\lfloor\frac{(2 i-2) m+1}{3}\right\rfloor-\left\lfloor\frac{2 i m+1}{3}\right\rfloor & \text { if } 3 \leq i \leq\left\lfloor\frac{n+2}{2}\right\rfloor, j=m-1 \\
(2 n-2 i+2) m+2+m-j-2\left\lfloor\frac{(2 n-2 i+5) m+1}{3}\right\rfloor & \text { if }\left\lfloor\frac{n+2}{2}\right\rfloor+1 \leq i \leq n, 2 \leq j \leq m-2 \\
(2 n-2 i+2) m+3-\left\lfloor\frac{(2 n-2 i+5) m+1}{3}\right\rfloor-\left\lfloor\frac{(2 n-2 i+3) m+1}{3}\right\rfloor & \text { if }\left\lfloor\frac{n+2}{2}\right\rfloor+1 \leq i \leq n, j=m-1 .\end{cases}
\end{aligned}
$$

Now $\max \left\{\left\{l(v) \mid v \in V\left(P_{m, n}^{*}\right)\right\} \cup\left\{l(e) \mid e \in E\left(P_{m, n}^{*}\right)\right\}\right\}=\left\lceil\frac{m n+2}{3}\right\rceil$ and $l$ is a function from $V\left(P_{m, n}^{*}\right) \cup E\left(P_{m, n}^{*}\right)$ into $\left\{1,2, \ldots,\left\lceil\frac{m n+2}{3}\right\rceil\right\}$.

The weights of the edges are given by

$$
\begin{aligned}
& w\left(e_{i}\right)= \begin{cases}m+2 & \text { if } i=1 \\
(2 i-2) m+2 & \text { if } 2 \leq i \leq\left\lfloor\frac{n+2}{2}\right\rfloor \\
(2 n-2 i+3) m+2 & \text { if }\left\lfloor\frac{n+2}{2}\right\rfloor+1 \leq i \leq n,\end{cases} \\
& w\left(e_{i j}\right)= \begin{cases}j+2 & \text { if } i=1,1 \leq j \leq m-1 \\
(2 i-3) m+2+j & \text { if } 2 \leq i \leq\left\lfloor\frac{n+2}{2}\right\rfloor, 1 \leq j \leq m-1 \\
(2 n-2 i+2) m+2+m-j & \text { if }\left\lfloor\frac{n+2}{2}\right\rfloor+1 \leq i \leq n, 1 \leq j \leq m-1 .\end{cases}
\end{aligned}
$$

The weights of the edges of $E$ under total labeling $l$ form a set of consecutive integers from 3 to $n m+2$ and no two edges have the same weight. Hence tes $\left(P_{m, n}^{*}\right)=\left\lceil\frac{n m+2}{3}\right\rceil$.

Consider $n$ copies of the graph $C_{m}$ and label the vertices in the $i^{t h}$ copy of $C_{m}$ as $v_{i}, v_{i 1}, v_{i 2}, \ldots$, $v_{i(m-2)}, v_{i+1}$ for $1 \leq i \leq n$. Then using the labelings as in $P_{m, n}^{*}$, we get the following result.

Corollary 2.1. [2] For any integers $m \geq 3, n \geq 3$, tes $\left(n C_{m}\right)=\left\lceil\frac{n m+2}{3}\right\rceil$.

In the graph $P_{m, n}^{*} \cup P_{m, r}^{*}$, labeling the vertices in $P_{m, r}^{*}$ by

$$
\left\{v_{i} \mid n+1 \leq i \leq n+r\right\} \cup\left\{v_{i j} \mid n+1 \leq i \leq n+r-1,1 \leq j \leq m-2\right\},
$$

we obtain the following result. 
Corollary 2.2. For any integers $m \geq 3, n \geq 3$, tes $\left(P_{m, n}^{*} \cup P_{m, r}^{*}\right)=\left\lceil\frac{m(n+r)+2}{3}\right\rceil$.

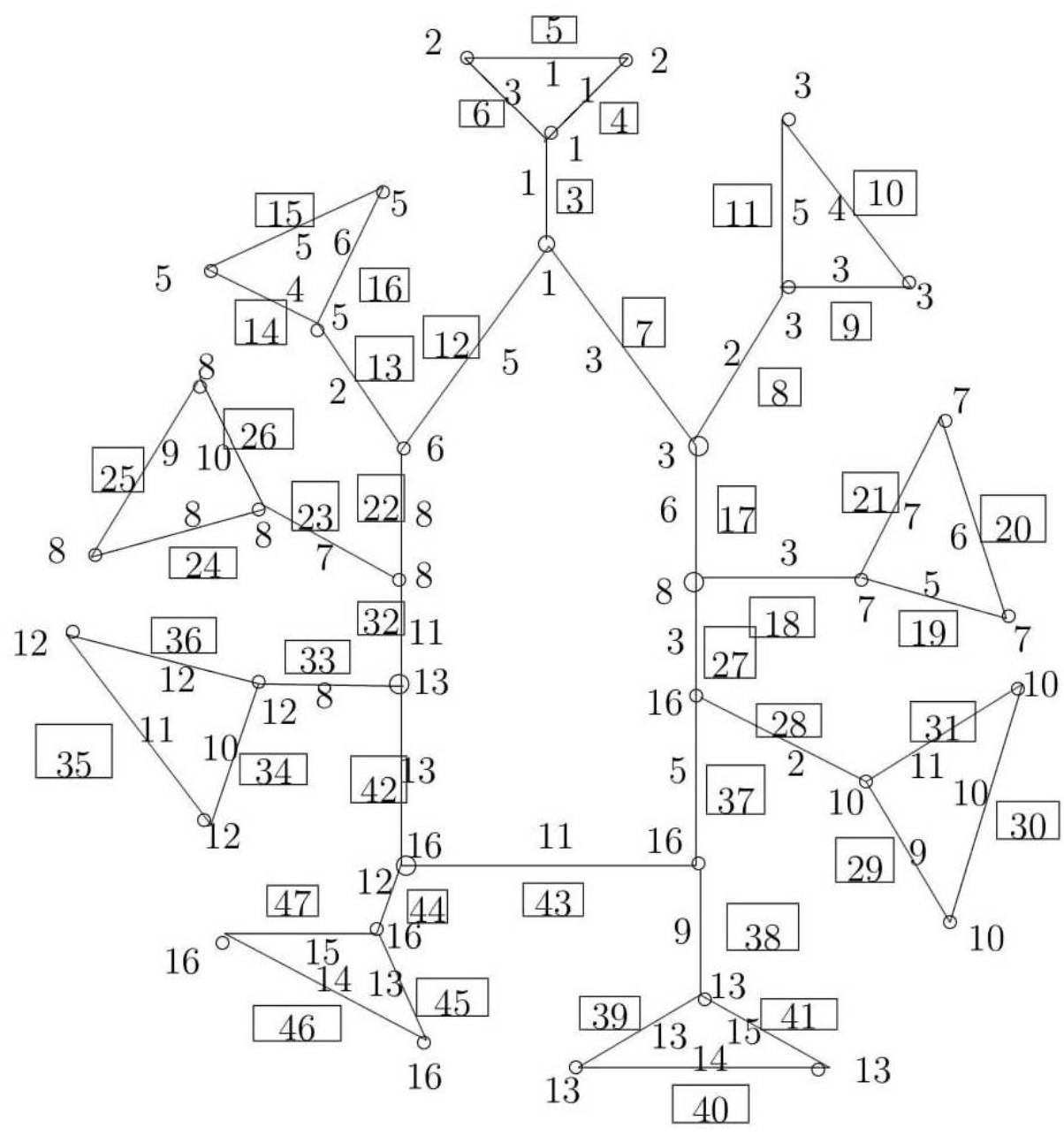

Figure 1. $C_{3} @ C_{9}$

\section{Open Problem}

In $C_{m} @ C_{n}$, we take $n$ copies of $C_{m}$. Instead of considering same cycles, consider $n$ cycles with different lengths $m_{1}, m_{2}, \ldots, m_{n}$ and denote the new graph by $C^{\prime}$. Prove that

$$
\operatorname{tes}\left(C^{\prime}\right)=\left\lceil\frac{\sum_{i=1}^{n} m_{i}+2}{3}\right\rceil \text {. }
$$

\section{Acknowledgement}

We thank anonymous reviewer for the valuable comments on an earlier version of the manuscript. 


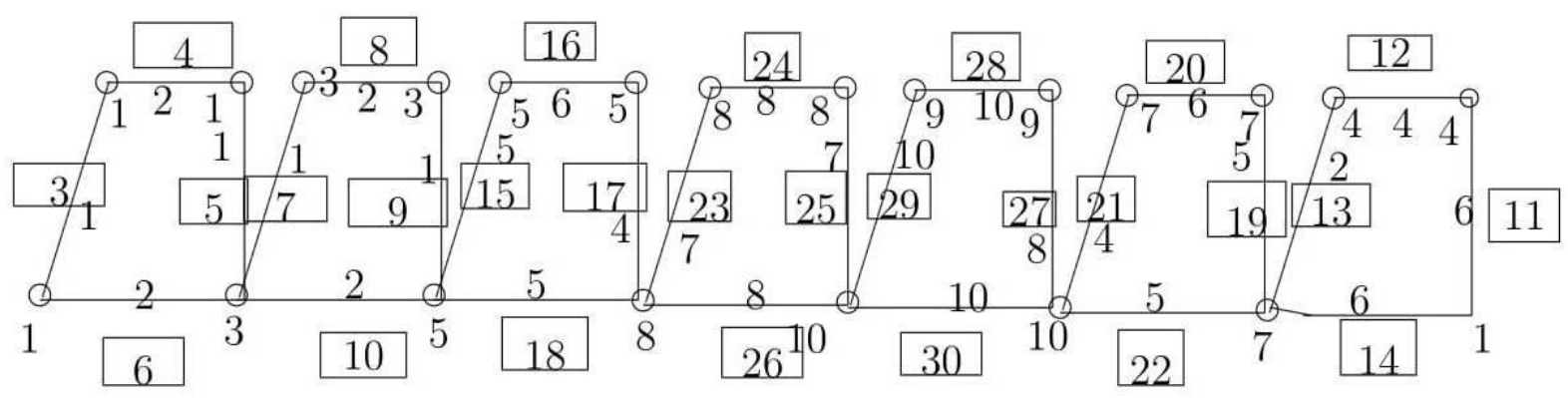

Figure 2. $P_{4,7}^{*}$

\section{References}

[1] A. Ahmad and M. Baca, Edge irregular total labeling of certain family of graphs, AKCE J.Graphs Combin., 6(1) (2009), 21-29.

[2] A. Ahmad, M. Baca and M.K. Siddiqui, Irregular total labeling of disjoint union of prisms and cycles, Australas J.Combin., 59(1) (2014), 98-106.

[3] M. Baca, S. Jendrol, M. Miller, and J. Ryan, On irregular total labeling, Discrete Math., 307 (2007), 1378-1388.

[4] J.A. Gallian, A dynamic survey of graph labeling, The Electron. J. Combin., (2018), \#DS6.

[5] R. Indra and T.A. Santiago, Total edge irregularity strength of generalised uniform theta graph, International Journal of Scientific Research, 7 (2018), 41-43.

[6] J. Ivanco and S. Jendrol, Total edge irregularity strength for trees, Discuss.Math.Graph Theory, 26 (2006), 449-456.

[7] S. Jendrol, J. Miskuf, and R. Sotak, Total edge irregularity strength of complete graphs and complete bipartite graphs, Discrete Math., 310 (2010), 400-407.

[8] P. Jeyanthi and A. Sudha, Total edge irregularity strength of disjoint union of double wheel graphs, Proyecciones J.Math., 35 (2016), 251-262.

[9] P. Jeyanthi and A. Sudha, On total edge irregularity strength of some graphs, Bulletin of the International Mathematical Virtual Institute, 9 (2019), 393-401.

[10] K. M. Kathiresan and R. Ramalakshmi, Total edge irregularity strength for three clases of graphs, Util. Math., 102 (2017), 321-329.

[11] S. Nurdin, A.N.M. Salman and E.T. Baskoro, The total edge-irregular strengths of the corona product of paths with some graphs, J. Combin. Math. Combin. Comput., 65 (2008), 163-175. 
[12] M.K. Siddiqui, On total edge irregularity strength of categorical product of cycle and path, AKCE J. Graphs. Combin., 9(1) (2012), 43-52. 